\title{
DESENVOLVIMENTO E CARACTERIZAÇÃO DE FILMES BIODEGRADÁVEIS DE FARINHA DE AMARANTO (Amaranthus caudatus)
}

\author{
C. H. PAGNO ${ }^{1}$, B. ALEXANDRE ${ }^{1}$, L. SCARTAZZINI ${ }^{2}$, A. R MONTEIRO ${ }^{2}$, A. O. RIOS ${ }^{1}$ e S. H. \\ FLÔRES ${ }^{1}$
}

${ }^{1}$ Universidade Federal do Rio Grande do Sul, Departamento de Ciência e Tecnologia de Alimentos

${ }^{2}$ Universidade Federal de Santa Catarina, Departamento de Engenharia Química e Engenharia de Alimentos

E-mail para contato: simone.flores@ufrgs.br

\begin{abstract}
RESUMO - O interesse em manter ou melhorar a qualidade dos produtos embalados e ao mesmo tempo reduzir o desperdício de embalagens, diminuindo o impacto ambiental causado por elas, tem encorajado a exploração de novas tecnologias para 0 desenvolvimento de embalagens. Dessa forma este trabalho tem por objetivo o desenvolvimento de filmes biodegradáveis a partir de farinha integral de amaranto e amido de amaranto. Os biofilmes foram produzidos pelo método de casting, onde foram avaliadas suas características mecânicas, solubilidade (S\%) e permeabilidade a vapor de água (PVA). Em relação a suas propriedades mecânicas de tração de ruptura (TS), percentagem de alongamento (E\%) e módulo de Young (MY) foi obtido para farinha integral valores de 4,82 \pm 0,54 MPa, 4,10 $\pm 1,17 \%$ e 290,68 \pm 61,80 MPa já para o filme de amido valores de 7,58 $\pm 1,30 \mathrm{MPa}, 2,96 \pm 0,37 \%$ e 341,34 $\pm 33,32 \mathrm{MPa}$, isso demonstra que os filmes de amido apesar de serem mais rígidos e resistentes, apresentar menos elasticidade. Em relação a PVA e solubilidade observou-se valores de 1,32 $\pm 0,20$ $10^{-10} \mathrm{~g} \cdot \mathrm{m}^{-1} \cdot \mathrm{s}^{-1} \cdot \mathrm{Pa}^{-1}$ e $40,31 \pm 2,12 \%$ para os filmes de farinha e valores de 5,36 $\pm 0,9310^{-}$ ${ }^{10} \mathrm{~g} \cdot \mathrm{m}^{-1} \cdot \mathrm{s}^{-1} \cdot \mathrm{Pa}^{-1} \mathrm{e} 49,53 \pm 3,10 \%$ para os filmes de amido. Tais resultados demonstram que tanto a farinhas de amaranto quanto seu amido extraído se mostram promissores como matéria prima para o desenvolvimento de filmes biodegradáveis apresentando propriedades mecânicas e PVA adequados para uso em alimentos.
\end{abstract}

\section{INTRODUÇÃO}

A principal função de uma embalagem é a proteção do alimento, evitando sua interação com o ambiente externo, prolongando sua conservação. No entanto a maioria das embalagens de alimentos é fabricado com polímeros não biodegradáveis desenvolvidos à base de petróleo e sua eliminação tem se tornado um grave problema ambiental. Dessa forma, a substituição parcial destes materiais com polímeros biodegradáveis a partir de fontes renováveis é uma alternativa, podendo reduzir o impacto da embalagem sobre o meio ambiente (De Camargo Andrade-Molina et al., 2013).

O interesse de manter, ou melhorar a qualidade dos produtos embalados e, ao mesmo tempo, 


\section{9 a 22 de outubro de 2014 \\ Florianópolis/SC}

reduzir o desperdício, tem encorajado a exploração de novos materiais para o desenvolvimento de embalagens, como os filmes biodegradáveis formulados com matérias-primas oriundas de recursos renováveis (Souza et al., 2012). O uso de biopolímeros agrícolas para o desenvolvimento de filmes comestíveis e/ou biodegradáveis pode ser uma alternativa para aumentar as suas aplicações e criar novos mercados, bem como a substituição de plásticos sintéticos.

Filmes biodegradáveis podem ser obtidos de diferentes fontes, como carboidratos e proteínas, as quais apresentam baixo custo e podem desempenhar um importante papel na conservação de alimentos. Dentre os carboidratos o uso de amido no preparo filmes é indicado como extremamente promissor nesse sentido, bem como o uso de farinha, que apresentam uma combinação de polissacarídeos, proteínas, lipídios tendo como vantagem que cada componente pode atribuir ao filme características especificas melhorando suas propriedades mecânicas ou de barreira (Dias et al., 2010).

O amaranto, um pseudocereal de rápido crescimento, com alta tolerância a condições áridas e solos pobres em que os cereais tradicionais não podem ser cultivados, suas sementes apresentam proporção significativa de proteínas (14 a $17 \%$ ), gordura (5 a $9 \%$ ) e amido (62 \%), que fez a sua farinha um material promissor para a formação do filme (Tapia-Blacido, et al., 2005). Dessa forma este trabalho teve por objetivo o desenvolvimento de filmes biodegradáveis a partir de amido e farinhas de Amaranto e avaliar suas propriedades mecânicas e propriedades de barreira a água.

\section{MATÉRIAS E MÉTODOS}

\subsection{Matéria Prima}

Para o desenvolvimento dos filmes foram utilizados farinha de Amaranto, obtida pela trituração dos grãos de Amaranto (Amaranthus caudatus), em moinho de grãos (Arbel, model MGR90, Brazil). A farinha resultante foi passada através de peneiras com granulometria de 150 mesh, o que passou pelos crivos foi utilizado para elaboração dos filmes. O amido foi obtido a partir da farinha de acordo com metodologia descrita por Araujo-Farro et al., (2010). Como agente plastificante foi utilizado glicerol (Merck).

\subsection{Preparo dos Filmes}

Os filmes foram formulados pelo método de casting, como descrito por Tapia-Blacido, et al., (2005) que consiste na elaboração de uma solução filmogênica, posteriormente seca sobre suportes de acrílico em estufa com circulação de ar. Para os filmes de farinha a solução filmogênica foi preparado com uma suspensão de $4 \%$ de farinha de amaranto (4 g / $100 \mathrm{~g}$ de solução filmogênica), e para os filmes de amido a solução filmogênica foi preparado com umas suspensão de 4 \% de amido (4 g / 100 $\mathrm{g}$ de solução filmogênica). As soluções foram homogeneizadas durante $15 \mathrm{~min}$. As soluções foram em seguida, aquecidas a temperaturas de processamento $\left(82^{\circ} \mathrm{C}\right.$ ), com agitação suave durante $30 \mathrm{~min}$. em banho maria ( DeLeo B450), após adicionou-se o plastificante (1\% v / v de solução filmogênica/filme) e a solução foi agitada durante mais 15 min. Em seguida a solução filmogênica foi vertida uniformemente sobre placas de acrílico circulares, na quantidade de $0,24 \mathrm{~g} / \mathrm{cm}^{2}$ da solução filmogênica. As películas foram secas em estufa com circulação forçada de ar (DeLeo B5AFD, 
Brasil ) a $35^{\circ} \mathrm{C}$ durante 16 horas

\subsection{Propriedades Mecânicas}

A propriedades mecânicas avaliadas foram Tração de Ruptura (TS), Percentagem de Alongamento $(\mathrm{E} \%)$ e Modulo de Yong (YM). Inicialmente os filmes foram cortados em tiras retangulares ( $80 \mathrm{~mm}-25 \mathrm{~mm}$ ) e armazenadas em cuba com umidade controlada a $58 \%$ por 48 horas. As análises foram realizadas em Texturômetro (TA.XT2i e Stable Micro Systems, UK) com uma célula de carga de $5 \mathrm{~kg}$, de acordo com ASTM D882-09 (2009). Dez repetições de cada filme foram analisados, utilizando uma distância inicial entre as garras de $50 \mathrm{~mm}$ e velocidade de elevação de 0,8 $\mathrm{mm} \cdot \mathrm{s}^{-1}$.

\subsection{Solubilidade}

A solubilidade filmes foi determinada em triplicata, com amostras dos filmes recortadas em discos de $2 \mathrm{~cm}$ de diâmetro. A porcentagem inicial da matéria seca de cada amostra foi determinada em estufa a $105^{\circ} \mathrm{C}$ por 24 horas. Após a primeira pesagem, as amostras foram imersas em recipientes contendo $30 \mathrm{~mL}$ de água destilada, e agitadas lenta e periodicamente por 24 horas e a $25^{\circ} \mathrm{C}$. Após este período, as amostras foram removidas e secas $\left(105^{\circ} \mathrm{C}\right.$ por 24 horas) para determinar a massa da matéria seca que não se dissolveu em água. A solubilidade foi calculado usando a Equação 1.

\section{Eq.(1) $S=[(w i-w f) / w i] .100$.}

Onde: onde wi é o peso seco inicial da amostra (g), e wf é o peso seco final da amostra após solubilização $(\mathrm{g})$.

\subsection{Permeabilidade a Vapor de Água (PVA)}

O PVA foi determinado gravimetricamente como descrito por Mei et al., (2013). As amostras foram colocadas em células de permeação (diâmetro interno $=63 \mathrm{~mm}$, altura $=25 \mathrm{~mm}$ ) carregada com cloreto de cálcio anidro e hermeticamente selada. As células de permeação foram colocadas numa câmara de vidro com solução de cloreto de sódio saturado para obter gradientes RH de 0/75\% a $25^{\circ} \mathrm{C}$. O ganho de peso foi determinada por pesagem da célula de permeação em balança analítica (AY 220, Shimadzu) com intervalos de $1 \mathrm{~h}$ durante o primeiro período 12 horas e após $24 \mathrm{~h}$. A permeabilidade ao vapor de água das amostras foi determinada em triplicado utilizando a Equação 2.

Eq. (2) $P V A=$ w.L/A.t. $\triangle p$

Onde: w é o peso da água que permeou através da película (g), L é a espessura da película, A é a área de permeação $\left(\mathrm{m}^{2}\right)$, $\mathrm{t}$ é o tempo de permeação, e $\triangle \mathrm{p}$ é a diferença de pressão de vapor de água entre os dois lados da película. 


\section{RESULTADOS}

Os resultados da propriedade mecânica de Tração de Ruptura (TS), Percentagem de Alongamento (E\%) e Modulo de Young (MY) dos filmes de farinhas e amido de amaranto pode ser observados na Tabela 1. Nota-se que em relação a TS, valor mais elevado foi obtido para o filme elaborado a partir do amido $(7,58 \pm 1,30 \mathrm{MPa})$, quando comparado ao filme elaborado com farinha (4,82 \pm 0,54 MPa). De acordo com Dias et al., (2010), a menor resistência dos filmes elaborados com farinhas, pode ser explicado tanto pela existência de irregularidades na microestrutura do filme formado, como pela presença de lipídios na farinha, que se alojam entre a as cadeias do polímero, diminuindo a continuidade da matriz formadora do filme. No entanto, o filme elaborado a partir da farinha, apesar de ser mais frágil, apresentou percentagem de alongamento mais elevado $(4,10 \pm 1,17$ $\%$ ), valor este $27 \%$ superior ao obtido pelo filme elaborado com amido. A maior rigidez do filme de amido também pode ser evidenciado pelo valor mais elevado do MY.

O filme elaborado com farinha de amaranto apresentou TS de 4,82 \pm 0,54 MPa, valor este superior ao obtido por outros autores ao avaliarem as propriedades mecânicas de biofilmes desenvolvidos com farinha de amaranto estudados por Tapia-Blacido, et al.,( 2005) (TS = 1,45 $\pm 0,04$ e farinha de amaranto sem fibras ( $\mathrm{TS}=2,1 \pm 0,3 \mathrm{MPa}$ ) (Tapia-Blacido, et al., 2011). No entanto, em relação a percentagem de alongamento o valor obtido neste trabalho de 4,10 $\pm 1,17 \%$, foi inferior aos obtidos na literatura para filme de farinhas de amaranto ( $\mathrm{E} \%=83,7 \pm 5,1 \%$ ) (Tapia-Blacido, et al., $2005)$ e farinha de amaranto sem fibras $(\mathrm{E} \%=51,9 \pm 3,6 \%)$ (Tapia-Blacido, et al., 2011). Em relação ao YM Tapia-Blacido, et al., (2005) obtiveram para seus filmes de farinha de amaranto valor de 215.0 $\pm 1.4 \mathrm{MPa}$, inferior ao obtidos neste trabalho de 290,68 $\pm 61,80 \mathrm{MPa}$, isso demonstra que o filme de farinha de amaranto obtido neste trabalho apresentou maior rigidez.

Tabela 1 - Propriedades mecânicas de Tração de Ruptura (TS), Percentagem de Alongamento (E\%) e Modulo de Young (MY), permeabilidade a vapor de água (PVA) e percentual de solubilidade (S\%) dos filmes elaborados com farinhas e amido de Amaranto

\begin{tabular}{rcc} 
& Farinha de Amaranto & Amido de Amaranto \\
\hline $\mathrm{TS}(\mathrm{MPa})$ & $4,82 \pm 0,54$ & $7,58 \pm 1,30$ \\
$\mathrm{E} \%$ & $4,10 \pm 1,17$ & $2,96 \pm 0,37$ \\
$\mathrm{MY}(\mathrm{MPa})$ & $290,68 \pm 61,80$ & $341,34 \pm 33,32$ \\
$\mathrm{PVA}\left(10^{-10} \mathrm{~g} \cdot \mathrm{m}^{-1} \cdot \mathrm{s}^{-1} \cdot \mathrm{Pa}^{-1}\right)$ & $1,32 \pm 0,20$ & $5,36 \pm 0,93$ \\
Solubilidade $(\%)$ & $40,31 \pm 2,12$ & $49,53 \pm 3,10$ \\
\hline
\end{tabular}

O filme elaborado com amido de amaranto apresentou TS de 7,58 \pm 1,30 $\mathrm{MPa}$, valor esse superior ao obtido para filmes de amido de quinoa estudados por Araujo-Farro et al., 2010 (TS = 7,56 $\pm 0,95 \mathrm{MPa}$ ) Entretanto o filme deste estudo apresentou valor similar aos obtidos para filmes de amido de mandioca (TS $=8 \mathrm{MPa}$ ) obtidos por Müller, et al., (2009). Em relação a percentagem de 


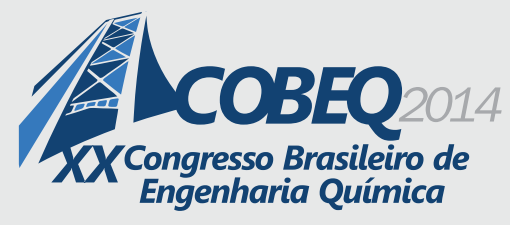

19 a 22 de outubro de 2014
Florianópolis/SC

alongamento o filme de amido de amaranto apresentou valor de 2,96 $\pm 0,37 \%$, valor este inferior ao obtido na literatura para amidos de outras fontes como amido de quinoa (E\% = 58,14 $\pm 3,16 \%$ ) (Araujo-Farro et al., 2010) e amido de mandioca $(\mathrm{E} \%=5,1 \pm 1,9 \%)$ (Alves et al., 2007).

Em relação a permeabilidade a vapor de água (PVA) dos filmes (Tabela 1), menor permeabilidade pode ser observado para os filmes elaborados com farinhas de amaranto (1,32 $\pm 0,20$ $\left.10^{-10} \mathrm{~g} \cdot \mathrm{m}^{-1} \cdot \mathrm{s}^{-1} \cdot \mathrm{Pa}^{-1}\right)$ em relação ao filme elaborado com amido de amaranto $\left(5,36 \pm 0,9310^{-10} \mathrm{~g} \cdot \mathrm{m}^{-1} \cdot \mathrm{s}^{-}\right.$ $\left.{ }^{1} \cdot \mathrm{Pa}^{-1}\right)$. Essa menor solubilidade se deve principalmente aos lipídios presentes na farinha, o qual aumenta a característica hidrofóbica do filme diminuindo assim sua interação com a água. Comportamento similar pode ser observado para o percentual de solubilidade, onde os filmes elaborados com farinha de amaranto $(40,31 \pm 2,12 \%)$ apresentaram solubilidade menor quando comparado com a solubilidade do filme elaborado com amido de amaranto (49,53 $\pm 3,10 \%)$. Ao desenvolver filmes de farinha de amaranto, Tapia-Blacido et al., (2007) obtiveram PVA de 0,8 $\pm 0,2$ $10^{-10} \mathrm{~g} \cdot \mathrm{m}^{-1} \cdot \mathrm{s}^{-1} \cdot \mathrm{Pa}^{-1}$ e percentual de solubilidade de 42,2 $\pm 1,8 \%$, já Tapia-Blacido, et al., (2011) obtiveram PVA igual a 3,8 $\pm 0,210^{-10} \mathrm{~g} \cdot \mathrm{m}^{-1} \cdot \mathrm{s}^{-1} \cdot \mathrm{Pa}^{-1}$ e solubilidade de 41,9 $\pm 2,4 \%$ para seus filmes elaborados com farinha de amaranto.

\section{CONCLUSÃO}

Tanto a farinha de amaranto como seu amido extraído, apresentam-se como uma boa alternativa de material prima renovável para elaboração de filmes biodegradáveis. $\mathrm{O}$ filme elaborado com amido de apresentou melhor característica mecânica de tração de ruptura, no entanto, apresenta baixa elasticidade ao ser comparado com o filme elaborado com farinha, dando origem a filmes mais rígidos. Apesar disso, da baixa resistência do filme elaborado com farinha de amaranto, o mesmo apresenta melhor característica de barreira e menor solubilidade, o que favorece seu uso em alimentos com teor de umidade mais elevado.

\section{REFERÊNCIAS}

ALVES, V. D. et al. Effect of glycerol and amylose enrichment on cassava starch film properties. Journal of Food Engineering, v. 78, n. 3, p. 941-946, 2// 2007.

ARAUJO-FARRO, P. C. et al. Development of films based on quinoa (Chenopodium quinoa, Willdenow) starch. Carbohydrate Polymers, v. 81, n. 4, p. 839-848, 7/23/ 2010.

DE CAMARGO ANDRADE-MOLINA, T. P. et al. Active biodegradable packaging for fresh pasta. LWT - Food Science and Technology, v. 54, n. 1, p. 25-29, 11// 2013.

DIAS, A. B. et al. Biodegradable films based on rice starch and rice flour. Journal of Cereal Science, v. 51, n. 2, p. 213-219, Mar 2010.

MEI, J. et al. Characterization of edible starch-chitosan film and its application in the storage of Mongolian cheese. International Journal of Biological Macromolecules, v. 57, n. 0, p. 1721, 6// 2013.

MÜLLER, C. M. O.; LAURINDO, J. B.; YAMASHITA, F. Effect of cellulose fibers on the crystallinity and mechanical properties of starch-based films at different relative humidity values. Carbohydrate Polymers, v. 77, n. 2, p. 293-299, 6/10/ 2009. 
PELISSARI, F. M. et al. Comparative study on the properties of flour and starch films of plantain bananas (Musa paradisiaca). Food Hydrocolloids, v. 30, n. 2, p. 681-690, 3// 2013.

TAPIA-BLACIDO, D. et al. Contribution of the starch, protein, and lipid fractions to the physical, thermal, and structural properties of amaranth (Amaranthus caudatus) flour films. Journal of Food Science, v. 72, n. 5, p. E293-E300, Jun-Jul 2007.

TAPIA-BLACIDO, D.; SOBRAL, P. J.; MENEGALLI, F. C. Development and characterization of biofilms based on Amaranth flour (Amaranthus caudatus). Journal of Food Engineering, v. 67, n. 1-2, p. 215-223, Mar 2005.

TAPIA-BLACIDO, D. R.; SOBRAL, P. J. D.; MENEGALLI, F. C. Optimization of amaranth flour films plasticized with glycerol and sorbitol by multi-response analysis. Lwt-Food Science and Technology, v. 44, n. 8, p. 1731-1738, Oct 2011. 